\title{
Demonstration of Self-Pulsating InP-on-Si DFB Laser Diodes
}

\author{
M. Shahin ${ }^{1,3, *}$ K. Ma ${ }^{2, *}$, A. Abbasi1, ${ }^{1,3}$, G. Roelkens ${ }^{1,3}$, G. Morthier ${ }^{1,3}$ \\ ${ }^{1}$ Photonics Research Group, Department of Information Technology, Ghent University-imec, Ghent, Belgium \\ ${ }^{2}$ Centre for Optical and Electromagnetic Research, State Key Laboratory for Modern Optical Instrumentation, Zhejiang Provincial Key \\ Laboratory for Sensing Technologies, Zhejiang University, Zijingang Campus, Hangzhou 310058, China
}

${ }^{3}$ Center for Nano- and Biophotonics (NB-Photonics), Ghent University, Ghent, Belgium

${ }^{*}$ Equal Contribution

Email: mahmoud.shahin@ugent.be

\begin{abstract}
Self-pulsating InP-on-Si two-section DFB laser diodes are demonstrated. The lasers have stable controllable pulsation frequencies at $12.5,25$ and $40 \mathrm{GHz}, \mathrm{RF}$ spectral widths of around $40 \mathrm{MHz}$ and $15 \mathrm{~dB}$ extinction ratio.
\end{abstract}

Index Terms - Integrated Optics Devices, Distributed Feedback Lasers, Semiconductor Lasers, Optical Devices, Silicon Photonics.

\section{INTRODUCTION}

As a result of the CMOS compatible fabrication technology and the low material cost, Silicon-on-Insulator (SOI) is emerging as the platform of choice for the implementation of photonic integrated circuits (PICs). However, as silicon is an indirect bandgap material, it cannot be used to make lasers. This motivates the integration of III-V materials on silicon to create laser diodes.

InP-on-SOI distributed feedback (DFB) laser diodes have already been used for various applications e.g. for high-speed direct modulation [1] and as tunable lasers [2]. The stable single mode operation, relatively simple structure and small footprint make DFB lasers attractive for many applications. In this paper, we discuss the implementation of self-pulsating III-V-on-SOI heterogeneously integrated DFB laser diodes. Such self-pulsating laser diodes can be used for the generation of optical microwave carriers or for the generation and/or extraction of optical clock signals. There are two mechanisms responsible for the self-pulsations. The first one is referred to as dispersive self-Q-switching [3], and it was first reported in [4]. This mechanism can result in self-pulsations at very high frequencies e.g. 64, 80 and $100 \mathrm{GHz}$ [5-6]. The second mechanism is caused by Longitudinal Spatial Hole Burning (LSHB) [7-9].

In this paper, different self-pulsating InP-on-SOI two-section DFB laser diodes are demonstrated, with pulsation frequencies of 12.5, 25 and $40 \mathrm{GHz}, \mathrm{RF}$ spectral widths of $40 \mathrm{MHz}$ and $15 \mathrm{~dB}$ extinction ratio.

\section{THEORY}

The theory behind both self-pulsation mechanisms of two-section DFB lasers is comprehensively explained in [6-11]. In the first mechanism, dispersive self-Q-switching, each laser section could be thought of as a stand-alone laser, with a different stop-band due to a different effective index $n_{e}$ in each section. The self-pulsation frequency is determined by the difference between the stopband width and the detuning of the Bragg wavelengths in both DFB sections [10]. If the stop-bands of both sections overlap, the lasing peak of one section coincides with the negative slope in the reflection spectrum of the other section, causing instability. This instability is manifesting itself in a variation of carrier and photon densities, which changes the effective indices, and shifts the stop-bands. The resulting power variation creates a periodic short-pulse train in the time domain. Intuitively this phenomenon results from the locking of the beating between two laser lines.
The second mechanism is caused by LSHB [7-9]. The introduction of carrier perturbation mechanisms throughout the laser, e.g. an asymmetrically-located $\lambda / 4$ phase shift in the case of DFB laser, causes asymmetry in the carrier distribution, which causes different effective indices and Bragg wavelengths across the laser. This perturbation is encouraged by biasing the sections at high and different currents. Multi-mode operation is a noticeable result of this phenomenon.
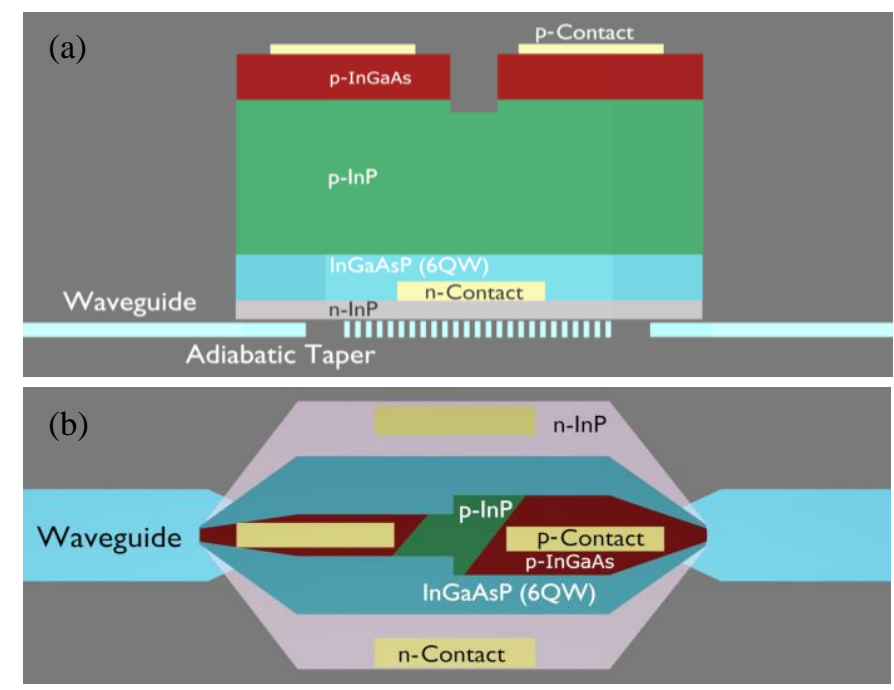

Fig. 1. a) Side-view of the demonstrated laser, with two electrically isolated sections having different widths, and b) top-view.

\section{DESIGN AND FABRICATION}

The III-V epitaxial stack for the self-pulsating DFB laser is adhesively-bonded [12] on the SOI using DVS-BCB (divinylsiloxane-bis-benzocyclobutene). The stack is patterned to resemble the structure in Fig.1. An adiabatic taper is used to couple the light from the III-V mesa to the silicon waveguide. In the center of the mesa, all the p-InGaAs and a small part of the p-InP is dry etched, to create two electrically isolated laser sections. This raises the resistance between the two p-contacts to more than $3000 \Omega$. The cut has an angle of $45^{\circ}$ to reduce reflections caused by the discontinuity in the top layer. The quantum well layer is wider than the mesa to reduce surface recombination. The fabrication process is comprehensively explained in [13-14].

We investigate two 500- $\mu \mathrm{m}$-long lasers, with different mesa width in each section. The different width creates different effective index $\left(n_{e}\right)$. This introduces a wavelength shift $\left(\Delta \lambda_{B}\right)$ of the stop-band of one section with respect to the other. Typical stop-band widths of similar lasers were shown to be around $4 \mathrm{~nm}$ [1]. Therefore, a $\Delta \lambda_{\mathrm{B}}$ of around $4 \mathrm{~nm}$ is desired to overlap the edges of the stop-bands. This requires a $\Delta \mathrm{n}_{\mathrm{e}}$ of around 0.01 . With the mesa width of one section being 4 $\mu \mathrm{m}$, we estimated the required mesa width in the other section for 
such $\Delta \mathrm{n}_{\mathrm{e}}$ to be between 2 and $2.5 \mu \mathrm{m}$. We therefore investigated two two-section DFB lasers (referred to as $\mathrm{L}_{1}$ and $\mathrm{L}_{2}$, respectively) with mesa widths being $2.5-4$ and $2-4 \mu \mathrm{m}$ respectively. There is also an asymmetrically-located $\lambda / 4$ phase-shift section in the left hand section of $\mathrm{L}_{2}$ to demonstrate LSHB-type self-pulsation.

\section{Characterization}

The lasers under study have a threshold current of around $2 \times 12$ $\mathrm{mA}$. To search for the self-pulsation peaks, a 2-dimentional current scan with $1 \mathrm{~mA}$ increment for both laser sections was performed. This unveiled stable peaks (moving in around $200 \mathrm{MHz}$ range due to temperature variations) with RF spectral widths of around $40 \mathrm{MHz}$, at $15{ }^{\circ} \mathrm{C}$. Controlling the current, and/or the temperature, shifts the peak over a few $\mathrm{GHz}$, before it vanishes. Fig.2. shows the selfpulsation at around 12.5, 25 and $40 \mathrm{GHz}$ measured using a Keysight N9010A EXA Electrical-Spectrum-Analyzer. The extinction ratio of the pulsations is around $15 \mathrm{~dB}$. The required current combinations (which are sensitive to fabrication variations) and the used laser are summarized in Table.1.

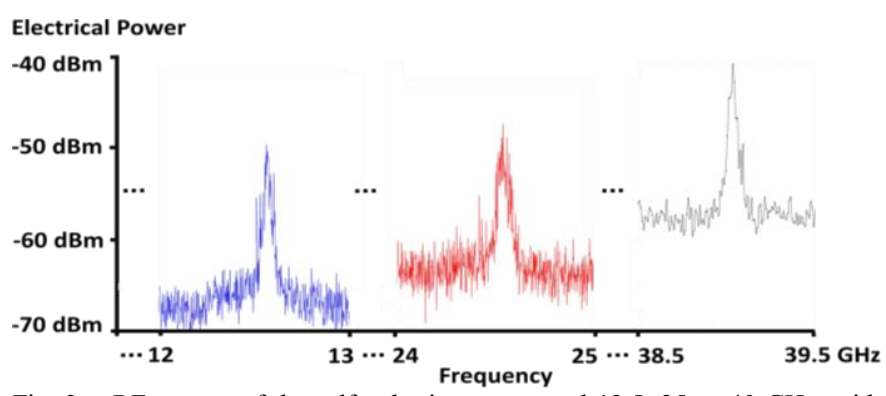

Fig. 2. RF spectra of the self-pulsations at around 12.5, 25 or $40 \mathrm{GHz}$, with spectral widths of around $40 \mathrm{MHz}$. The noise-floor level of the ESA increases for higher frequencies.

To demonstrate the stop-bands overlap, the optical spectrum at two bias current combinations in $\mathrm{L}_{1}$ is shown in Fig.3.a. One section is biased at the self-pulsation current (Table.1), while the other section is biased close to threshold. The stop-bands of both sections overlap. When biasing both sections at the correct combination, self-pulsation happens (Fig.3.b). Two peaks spaced by $0.31 \mathrm{~nm}$ are produced, which correspond to a self-pulsation at $38.75 \mathrm{GHz}$.

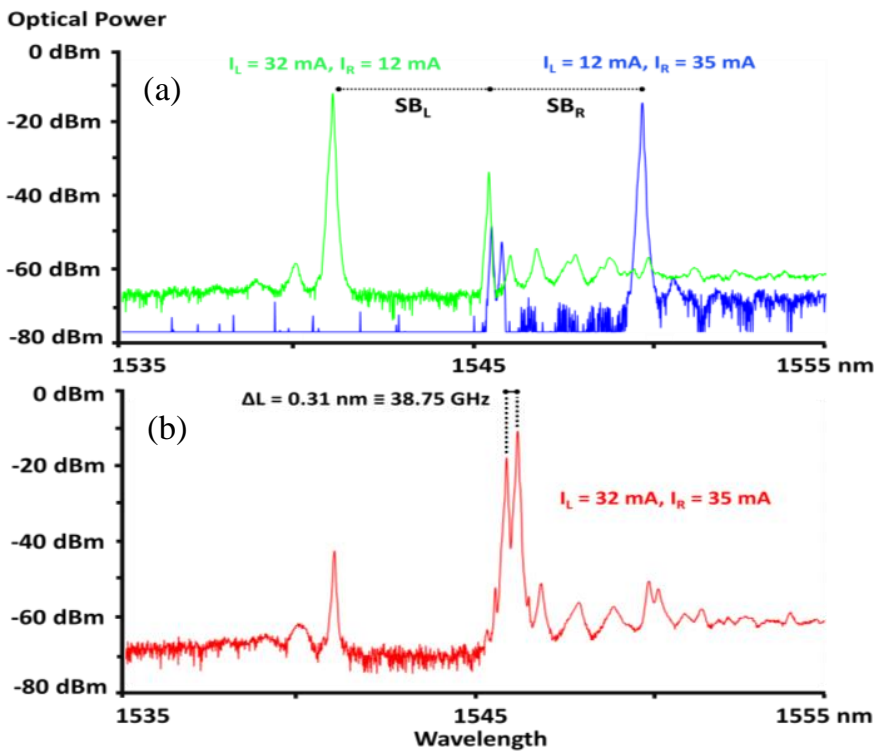

Fig. 3. a) An overlap of the stop-bands $\left(\mathrm{SB}_{\mathrm{L}}\right.$ and $\left.\mathrm{SB}_{\mathrm{R}}\right)$ is clear for each laser section when the other section is biased close to threshold, b) two peaks spaced by $0.31 \mathrm{~nm}$ are produced, which corresponds to $38.75 \mathrm{GHz}$.
Table.1: Summary of the current combinations to produce the self-pulsation

\begin{tabular}{|c|c|c|c|}
\hline Laser & Pulsation Frequency $(\mathrm{GHz})$ & $\mathrm{IL}_{\mathrm{L}}(\mathrm{mA})$ & $\mathrm{I}_{\mathrm{R}}(\mathrm{mA})$ \\
\hline $\mathrm{L}_{2}$ & 12.5 & 45 & 19 \\
\hline $\mathrm{L}_{2}$ & 24.5 & 43 & 23 \\
\hline $\mathrm{L}_{1}$ & 38.75 & 32 & 35 \\
\hline
\end{tabular}

In the same way, the optical spectrum corresponding to the 12.5 $\mathrm{GHz}$ pulsation is shown for $\mathrm{L}_{2}$ in Fig. 4 . We notice that the stopbands of the laser sections don't overlap. However, the self-pulsation is seen in the lasing-peak split of $0.096 \mathrm{~nm}$, which corresponds to 12.5 GHz. This is due to the carrier perturbation caused by the asymmetrically-located phase-shift, which creates different Bragg wavelengths across the laser. This instability and the observed multimode operation agrees with the explanation in [9].

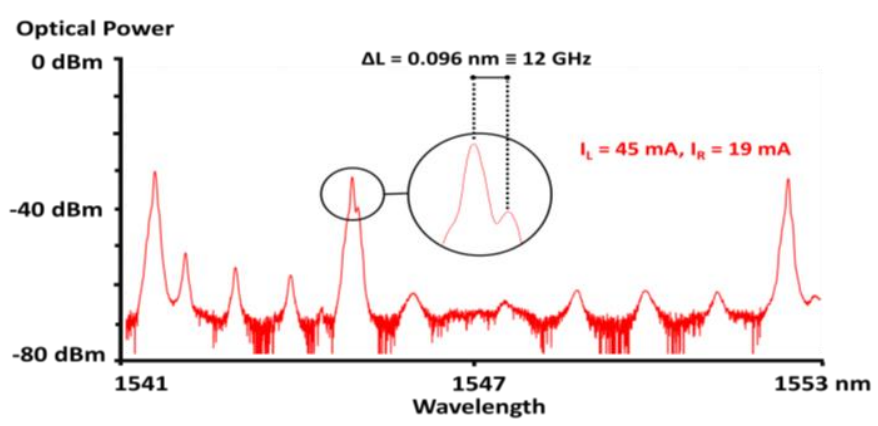

Fig. 4. Self-pulsation happens due to spatial hole burning, resulting in the split of two peaks spaced by $0.096 \mathrm{~nm}$, which corresponds to $12.5 \mathrm{GHz}$.

\section{CONCLUSION}

Self-pulsating InP-on-Si two-section DFB laser diodes, with a wide range of pulsation frequencies were demonstrated. The RF spectra have widths of $40 \mathrm{MHz}$, and an extinction ratio of $15 \mathrm{~dB}$. Choosing the pulsation frequency can be done by choosing the correct current values and/or controlling the temperature. Locking the pulsation frequency to a specific frequency by applying a small $\mathrm{RF}$-voltage is being investigated now.

\section{ACKNOWLEDGMENT}

The authors acknowledge the funding of the Methusalem program of the Flemish Government, and the international cooperation research and exchange program for doctoral students of Zhejiang University.

\section{REFERENCES}

[1] A. Abbasi et al., Optics Express, pp. 26479-26485, 23(20), (2015).

[2] S. Dhoore et al., Optics Letters, pp. 1121-1124, 42(6), (2017).

[3] B. Sartorius et al., IEEE JQE, pp. 211-218, 33(2), (1997).

[4] M. Mohrle et al., IEEE PTL, pp. 976-978, 4(9), (1992).

[5] B. Sartorius et al., IEEE JSTQE, pp. 535-538, 1(2), (1995).

[6] M. Mohrle et al., IEEE JQE, pp. 69-78, 32(1), (1996).

[7] A. Lowery, Electronics Letters, pp. 1852-1854, 29(21), (1993).

[8] D. Marcenac et al., Electronics Letters, pp. 1137-1138, 30(14), (1994).

[9] R. Schatz, IEEE JQE, pp. 1443-1449, 28(6), (1992).

[10] M. Mohrle et al., IEEE JSTQE, pp. 217-223, 7(2), (2002).

[11] U. Bandelow et al., IEEE PTL, pp. 1176-1179, 5(10), (1993).

[12] S. Keyvaninia et al., Optical Materials Express, pp. 35-46, 3(1), (2012).

[13] S. Keyvaninia et al., Optics Letters, pp. 5434-5437, 38(24), (2013).

[14] G. Roelkens et al., Photonics (invited), pp. 970-1004, 2(3), (2015). 